\title{
Association of low expression of E-cadherin and $\beta$-catenin with the progression of early stage human squamous cervical cancer
}

\author{
JING JIANG $^{1}$, XINLING LI ${ }^{1}$, XIANGMEI YIN ${ }^{1}$, JIEYING ZHANG $^{2}$ and BIN SHI ${ }^{1}$ \\ Departments of ${ }^{1}$ Obstetrics and Gynecology and ${ }^{2}$ Pathology, \\ The Second Hospital of Hebei Medical University, Shijiazhuang, Hebei 050000, P.R. China
}

Received February 10, 2018; Accepted December 21, 2018

DOI: $10.3892 /$ ol.2019.10266

\begin{abstract}
The precise involvement and mechanisms of human papilloma virus type 16 (HPV16) in epithelial-mesenchymal transition (EMT) of cervical intraepithelial neoplasia (CIN) and squamous cervical cancer (SCC) remain unknown. The present study aimed to examine the expression of EMT indicators and their association with HPV16 in CIN and early stage SCC, and their prognostic value in early stage SCC. The expression levels of E-cadherin, $\mathrm{N}$-cadherin, $\beta$-catenin, vimentin, and fibronectin were determined by immunohistochemistry in 40 patients with normal uterine cervix, 22 patients with CIN1, 60 patients with CIN2-3, and 86 patients with SCC, stage Ia-IIa, according to the International Federation of Gynecology and Obstetrics. The expression of the epithelial indicators E-cadherin and $\beta$-catenin gradually declined, and the mesenchymal indicators $\mathrm{N}$-cadherin, vimentin, and fibronectin increased with progression of the cervical lesions $(\mathrm{P}<0.05)$. Patients with $\mathrm{SCC}$ with lymph node metastasis, parametrial invasion, negative E-cadherin, and negative $\beta$-catenin expression had shorter overall survival $(\mathrm{P}=0.001$, $\mathrm{P}=0.015, \mathrm{P}=0.014$, and $\mathrm{P}=0.043$, respectively) and disease-free survival $(\mathrm{P}=0.002, \mathrm{P}=0.021, \mathrm{P}=0.025$, and $\mathrm{P}=0.045$, respectively) time. Multivariate survival analysis indicated that lymph node metastasis [Hazard ratio $(\mathrm{HR})=3.544 ; \mathrm{P}=0.010$ ], parametrial invasion $(\mathrm{HR}=2.014 ; \mathrm{P}=0.007)$ and $\mathrm{E}$-cadherin expression $(\mathrm{HR}=0.163 ; \mathrm{P}<0.001)$ were independently associated with overall survival, but also with disease-free survival $(\mathrm{HR}=3.612, \mathrm{P}=0.009 ; \mathrm{HR}=1.935, \mathrm{P}=0.011 ; \mathrm{HR}=0.168$, $\mathrm{P}<0.001$, respectively). In patients with CINs, HPV16 infection was negatively correlated with the expression of E-cadherin, and positively correlated with the expression of N-cadherin, vimentin, and fibronectin. EMT occurs during the progression
\end{abstract}

Correspondence to: Dr Jing Jiang, Department of Obstetrics and Gynecology, The Second Hospital of Hebei Medical University, 215 West Heping Road, Sijiazhuang, Hebei 050000, P.R. China E-mail: jiangjing011@sina.com

Key words: squamous cervical cancer, cervical intraepithelial neoplasia, precancerous lesions, epithelial-mesenchymal transition, HPV, prognosis of CINs to early stage SCC, and is associated with HPV16 infection in CINs. Lymph node metastasis and parametrial invasion are poor prognostic factors for SCC, while positive E-cadherin expression may serve as a protective prognostic factor for SCC.

\section{Introduction}

Cervical cancer (CC) is the third most common cancer in females worldwide, following breast and colon cancer (1). Squamous cervical cancer (SCC) is the major pathological type of $\mathrm{CC}$, which is believed to gradually develop from cervical intraepithelial neoplasia (CIN) (2). One of the causes of CC is persistent high-risk human papillomavirus (HPV) infection, and HPV type 16 (HPV16) in particular is associated with $55.2 \%$ of SCC cases (3). With widespread screening and improvements in diagnostic techniques for cervical precancerous lesions, the incidence of $\mathrm{CC}$ has decreased by $65 \%$ in certain Western countries over the past 40 years $(4,5)$. Nevertheless, invasion and metastasis of SCC remain major causes of postoperative relapse and mortality.

Tumor invasion and metastasis are a complex multistep process, involving multiple genes. Weakened adhesion and increased capacity for migration in tumor cells constitute a basis for tumor invasion and metastasis $(6,7)$. A number of studies have indicated that epithelial-mesenchymal transition (EMT) serves a role in tumor invasion and metastasis (8-11). In addition, studies have verified the important role of EMT in solid tumors, including ovarian, breast, prostate, lung, and liver cancer $(12,13)$. EMT was first proposed by Greenburg and Hay in 1982 (14) as the process by which epithelial cells lose their stable structure and their polarity and transform into freely migrating mesenchymal cells in the cell matrix, under certain special physiological or pathological conditions. The EMT process is characterized by the following: Reduction in the expression of or loss of cell adhesion molecules, including E-cadherin, transformation of the cytoskeleton from being majorly composed of epithelial cytokeratin to a cytoskeleton dominated by vimentin, loss of intercellular junctions, changes in cell morphology, and improved movement capacity (15). Markers of epithelial cell EMT, including E-cadherin and $\beta$-catenin, and mesenchymal cell markers, including vimentin and fibronectin, are used to study the biological behavior of EMT in tissues $(16,17)$. A number of studies have detected 
EMT in SCC and evidence from surgical specimens of SCC suggests that SCC progression is accompanied by E-cadherin downregulation and vimentin upregulation (18-21). HPV16 E6 or E7 have been indicated to regulate EMT in cervical epithelial cells, therefore, promoting tumor progression and metastasis $(22,23)$. Nevertheless, the precise involvement and mechanisms of HPV16 in the EMT of CIN and SCC lesions remain unknown.

In the present study, changes in the EMT indicators, including E-cadherin, $\beta$-catenin, $\mathrm{N}$-cadherin, vimentin, and fibronectin were studied in clinical tissue specimens, from low-grade CIN1 to high-grade CIN 2 and 3 (24), in addition to early stage SCC, in association with HPV16. The association among the EMT indicators and the clinicopathological features of patients with early stage SCC was also examined, and their prognostic value in SCC analyzed.

\section{Materials and methods}

Patient sample. Between January 2010 and December 2012, a total of 208 patients (mean age, $44.1 \pm 8.6$ years; range, 25-61 years) consulted at The Second Hospital of Hebei Medical University (Shijiazhuang, China) for abnormal bleeding or increased leucorrhea discharge. There were 86 cases of SCC, 82 cases of CIN, and 40 cases of leiomyoma with normal cervix. The patients who had received radiotherapy or chemotherapy were excluded. Among the 86 patients with SCC, 60 had stage Ia-b disease and 26 had stage IIa disease, according to the International Federation of Gynecology and Obstetrics (FIGO) 2000 standard (25). These patients underwent radical hysterectomy and pelvic lymph nodes resection, and the pathological examination confirmed the SCC in all 86 patients. Among them, 9 patients had parametrial invasion and 37 had lymph node metastasis. Among the 82 patients with CIN, 22 had CIN grade 1 and 60 had CIN grade 2-3 lesions according to the 2014 World Health Organization classification (26). Pathological examination performed at The Second Hospital of Hebei Medical University (Shijiazhuang, China) did not reveal any abnormal findings in the uterine cervix of the control cases, who underwent hysterectomy due to hysteromyoma.

The project was approved by the Institutional Review Board of The Second Hospital of Hebei Medical University (Shijiazhuang, China). Written informed consent was obtained from each patient prior to recruitment in the present study.

HPV diagnosis. Cervical exfoliated cells of all patients were clinically collected preoperatively using a cervical brush and were tested with the Cobas 4800 DNA HPV Test (Roche Molecular Systems, Pleasanton, CA, USA), according to the manufacturer's protocol. This test is a polymerase chain reaction fully automated method detecting separately HPV16, HPV18 and 12 other hrHPV types, including $31,33,35,39$, 45, 51, 52, 56, 58, 59, 66, and 68 (27). All the HPV-positive patients in the present study were HPV16-positive.

Immunostaining for EMT markers. According to the previously described method (28), paraffin cervical sections ( $4 \mu \mathrm{m})$ were immersed in xylene for $10 \mathrm{~min}$ twice at room temperature, and hydrated using a graded series of ethanol $(100,95,80$ and $70 \%$ ) all for $5 \mathrm{~min}$ at room temperature. Antigen retrieval was performed by immersing the sections in $0.01 \mathrm{M}$ citrated buffer (pH 6.0) in a pressure cooker and autoclaving for $15 \mathrm{~min}$. Endogenous peroxidase activity was blocked with $3 \% \mathrm{H}_{2} \mathrm{O}_{2}$ for $15 \mathrm{~min}$ at room temperature and then incubated with primary antibodies for $2 \mathrm{~h}$ at room temperature. Normal cervical tissues were used as the positive control and PBS was used instead of primary antibody as the negative control. Mouse anti-human E-cadherin (dilution, 1:100; cat. no. ab1416) N-cadherin (dilution, 1:500; cat. no. ab98952), $\beta$-catenin (dilution, 1:1,000; cat. no. ab22656), vimentin (dilution, 1:1,000; cat. no. ab8069), and fibronectin (dilution, 1:100; cat. no. ab6328) monoclonal antibodies all purchased from Abcam (Cambridge, MA, USA) were used with the streptavidin-peroxide (SP) kit and diaminobenzidine (DAB) chromogenic reagent kits (Beijing Zhongshan Biotechnology Co., Ltd., Beijing, China), according to the manufacturer's protocols. The sections were incubated with SP for $1 \mathrm{~h}$ at $37^{\circ} \mathrm{C}$ and then stained using DAB for $10 \mathrm{~min}$ at room temperature. Specimens were observed under a light microscope (magnification, x400) by two pathologists, at The Second Hospital of Hebei Medical University, separately using five high-power randomly selected fields for each patient. The percentage and staining intensity of cells positive for each of the aforementioned proteins were determined. The scores for the percentage of positive cells were: 0 , no positive cells; $1, \leq 25 \%$ positive cells; $2,26-50 \%$ positive cells; $3,51-75 \%$ positive cells; and $4,>75 \%$ positive cells (29). With regard to staining intensity, the scores were: 1 , weak; 2 , moderate; and 3 , strong. If the product of percentage and scoring intensity scores was $>3$, the specimen was considered to be immunohistochemically positive for the corresponding marker (30).

Statistical analysis. Continuous data were presented as mean \pm standard deviation and analyzed using one-way analysis of variance with the Tukey's post hoc test. Categorical data were presented as frequencies and analyzed using the $\chi^{2}$ test. The associations among HPV16 and E-cadherin, $\beta$-catenin, $\mathrm{N}$-cadherin, vimentin, and fibronectin were analyzed using Spearman's correlation coefficient. Survival curves were generated by the Kaplan-Meyer method and the survival rates were compared using the log-rank test. Univariate and multivariate survival analyses were performed using the Cox regression model for overall survival (OS) and disease-free survival (DFS) time. Data were analyzed using SPSS v.21.0 (IBM Corp., Armonk, NY, USA). Two-sided $\mathrm{P}<0.05$ values were considered to indicate a statistically significant difference.

\section{Results}

Characteristics of the patients. Table I presents the characteristics of the patients. Patients with CIN were significantly younger compared with the controls and patients with early stage SCC $(\mathrm{P}<0.001)$. The rate of HPV16 infection significantly gradually increased from the controls $(0 \%)$, to CIN1 (9.1\%), CIN2-3 (41.7\%), and SCC (94.2\%) ( $<<0.001)$.

Expression of E-cadherin, $\beta$-catenin, $N$-cadherin, vimentin, and fibronectin in cervical lesions. Normally, brown granules in the membrane of epithelial cells were considered to indicate positive expression for $\mathrm{E}$-cadherin, $\beta$-catenin, and $\mathrm{N}$-cadherin, while brown granules in the cytoplasm of extracellular matrix 
Table I. Clinical characteristics of the patients.

\begin{tabular}{|c|c|c|c|c|c|}
\hline Variables & Normal control & CIN1 & CIN2-3 & $\mathrm{SCC}$ & P-value \\
\hline No. & 40 & 22 & 60 & 86 & \\
\hline Age $($ mean $\pm \mathrm{SD})$ & $48.8 \pm 2.9$ & $33.5 \pm 6.5$ & $37.0 \pm 5.8$ & $49.6 \pm 5.9$ & $<0.001$ \\
\hline HPV16 infection (\%) & 0 & $9.1(2 / 22)$ & $41.7(25 / 60)$ & $94.2(81 / 86)$ & $<0.001$ \\
\hline \multicolumn{6}{|l|}{ FIGO stage } \\
\hline Ia-b & - & - & - & 60 & \\
\hline IIa & - & - & - & 26 & \\
\hline \multicolumn{6}{|l|}{${ }^{\text {aHistological grade }}$} \\
\hline G1 & - & - & - & 22 & \\
\hline $\mathrm{G} 2-3$ & - & - & - & 64 & \\
\hline \multicolumn{6}{|l|}{ Parametrial invasion } \\
\hline Yes & - & - & - & 9 & \\
\hline No & - & - & - & 77 & \\
\hline \multicolumn{6}{|l|}{ Lymph node metastasis } \\
\hline Yes & - & - & - & 37 & \\
\hline No & - & - & - & 49 & \\
\hline \multicolumn{6}{|l|}{ Recurrence } \\
\hline Yes & - & - & - & 23 & \\
\hline No & - & - & - & 63 & \\
\hline
\end{tabular}

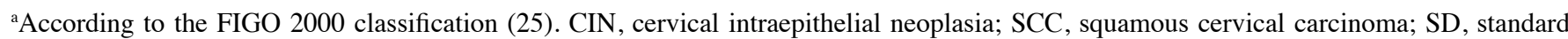
deviation; FIGO, International Federation of Gynecology and Obstetrics; -, not available; HPV16, human papilloma virus type 16.

were considered to indicate positive expression for vimentin and fibronectin $(20,31,32)$. E-cadherin expression was the highest in normal cervical tissue, and decreased with disease progression in the order of CIN1, CIN2-3, and SCC, respectively (Fig. 1). $\beta$-catenin indicated abnormal expression in the CIN/SCC cases in the form of weakened membranous staining and increased cytoplasmic staining (Fig. 2). Similar to E-cadherin expression, the percentage of patients with positive $\beta$-catenin expression decreased with disease progression. The expression of $\mathrm{N}$-cadherin (Fig. 3), vimentin (Fig. 4), and fibronectin (Fig. 5) exhibited an opposite trend, therefore, positive staining rate increased with disease progression. The expression of all five proteins was not significantly different between the control and CIN1 groups. The expression of E-cadherin, $\beta$-catenin, and vimentin was significantly different $(\mathrm{P}=0.024-0.026)$, but the expression of $\mathrm{N}$-cadherin and fibronectin was not significantly different between the CIN1 and CIN2-3 groups. However, the differences were significant for comparisons among all the other groups $(\mathrm{P} \leq 0.002)$.

Clinicopathological features and prognostic values of EMT indicators. During the median follow-up of 60 months (range, 10-90 months), 23/86 (26.7\%) patients had a SCC recurrence. Among those 23 patients, 20 (87.0\%) succumbed to cancer progression. The 5-year DFS and OS rates were 73.3 and $76.7 \%$, respectively.

Patients with SCC with negative E-cadherin and negative $\beta$-catenin expression had significantly shorter OS times $(\mathrm{P}=0.014$ and $\mathrm{P}=0.043$, respectively) and shorter DFS times $(\mathrm{P}=0.025$, and $\mathrm{P}=0.045$, respectively) (Fig. 6). Patients with
SCC with lymph node metastasis and parametrial invasion also had significantly shorter $\mathrm{OS}$ times $(\mathrm{P}=0.001$ and $\mathrm{P}=0.015$, respectively) and shorter DFS times $(\mathrm{P}=0.002$ and $\mathrm{P}=0.021$, respectively) (Fig. 7). The other clinical features, including age, FIGO stage, and histological grade, and the other three EMT indicators, including N-cadherin, vimentin, and fibronectin indicated no significant differences.

Among patients with SCC, univariate survival analyses revealed that SCC with lymph node metastasis, parametrial invasion, negative E-cadherin, and negative $\beta$-catenin were correlated with shorter $\mathrm{OS}(\mathrm{P}=0.003, \mathrm{P}=0.022, \mathrm{P}<0.001$, and $\mathrm{P}<0.001$, respectively; Table II) and DFS $(\mathrm{P}=0.004, \mathrm{P}=0.030$, $\mathrm{P}<0.001$, and $\mathrm{P}<0.001$, respectively; Table II).

The multivariate analysis indicated that among patients with SCC, lymph node metastasis, parametrial invasion, and negative E-cadherin expression were independent prognostic predicators for $\mathrm{OS}(\mathrm{P}=0.010, \mathrm{P}=0.007$ and $\mathrm{P}<0.001$, respectively; Table III) and DFS $(\mathrm{P}=0.009, \mathrm{P}=0.011$ and $\mathrm{P}<0.001$, respectively; Table III).

Correlation of HPV16 infection and EMT proteins. The Cobas $4800 \mathrm{HPV}$ test indicated that no case in the control group was HPV16 positive, and 27/82 (32.9\%) patients with CINs had HPV16 infection. Correlation analysis indicated that the expression rate of $\beta$-catenin was not significantly correlated to HPV16 infection $(\mathrm{P}=0.941)$, while the expression rates of E-cadherin, N-cadherin, vimentin and fibronectin were significantly correlated to HPV16 infection ( $\mathrm{P} \leq 0.001$; Table IV). E-cadherin had a negative relationship with HPV16 infection $\left(r_{s}=-0.424\right)$, while N-cadherin, vimentin, and fibronectin had 

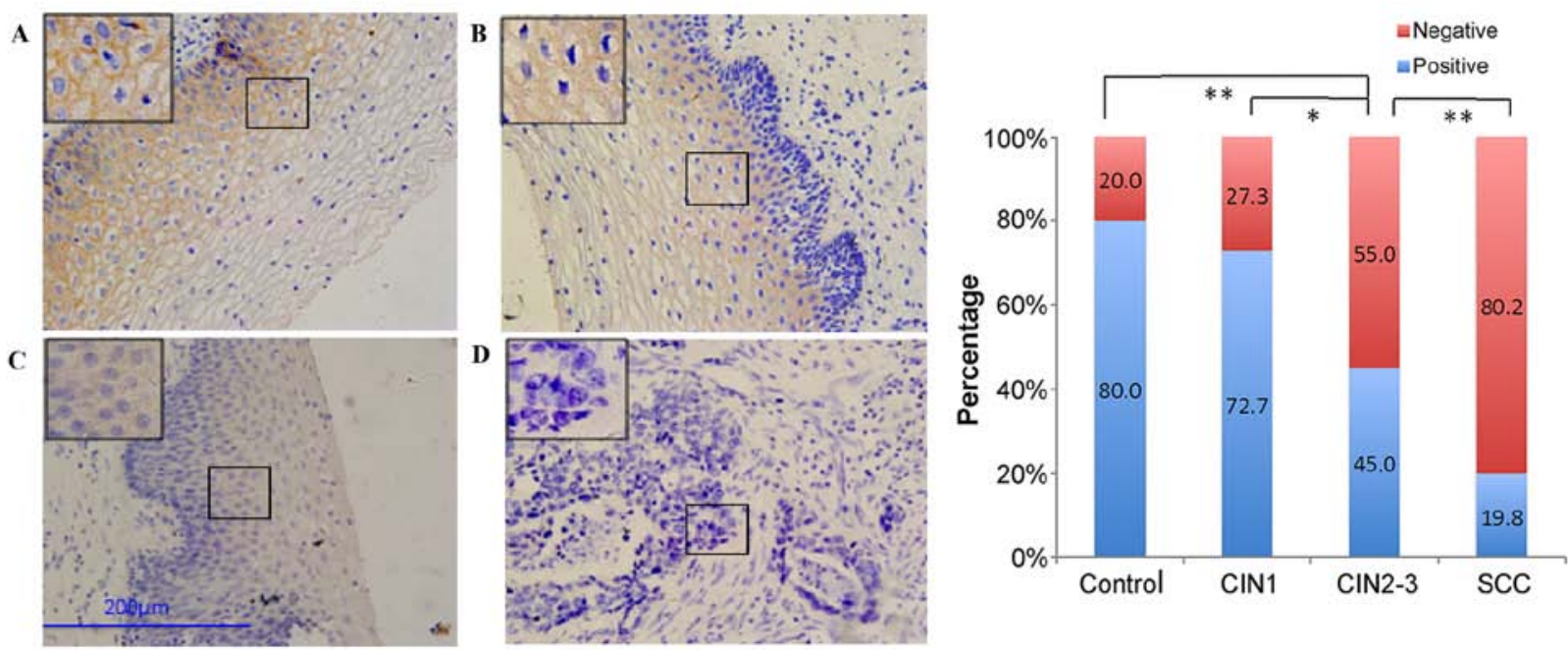

Figure 1. Immunohistochemical staining of E-cadherin in cervical sections. Immunohistochemical staining of E-cadherin in (A) normal human cervical tissue and (B) CIN1 (strong membranous staining), (C) CIN2-3 (moderate staining), and (D) SCC (weak staining). Magnification, x400. The scale bar is given at the left bottom. Insets are magnified images from selected areas (small squares). ${ }^{*} \mathrm{P}<0.05,{ }^{* *} \mathrm{P}<0.01$. CIN1/2-3, cervical intraepithelial neoplasia grade $1 / 2-3$; SCC, squamous cervical carcinoma.
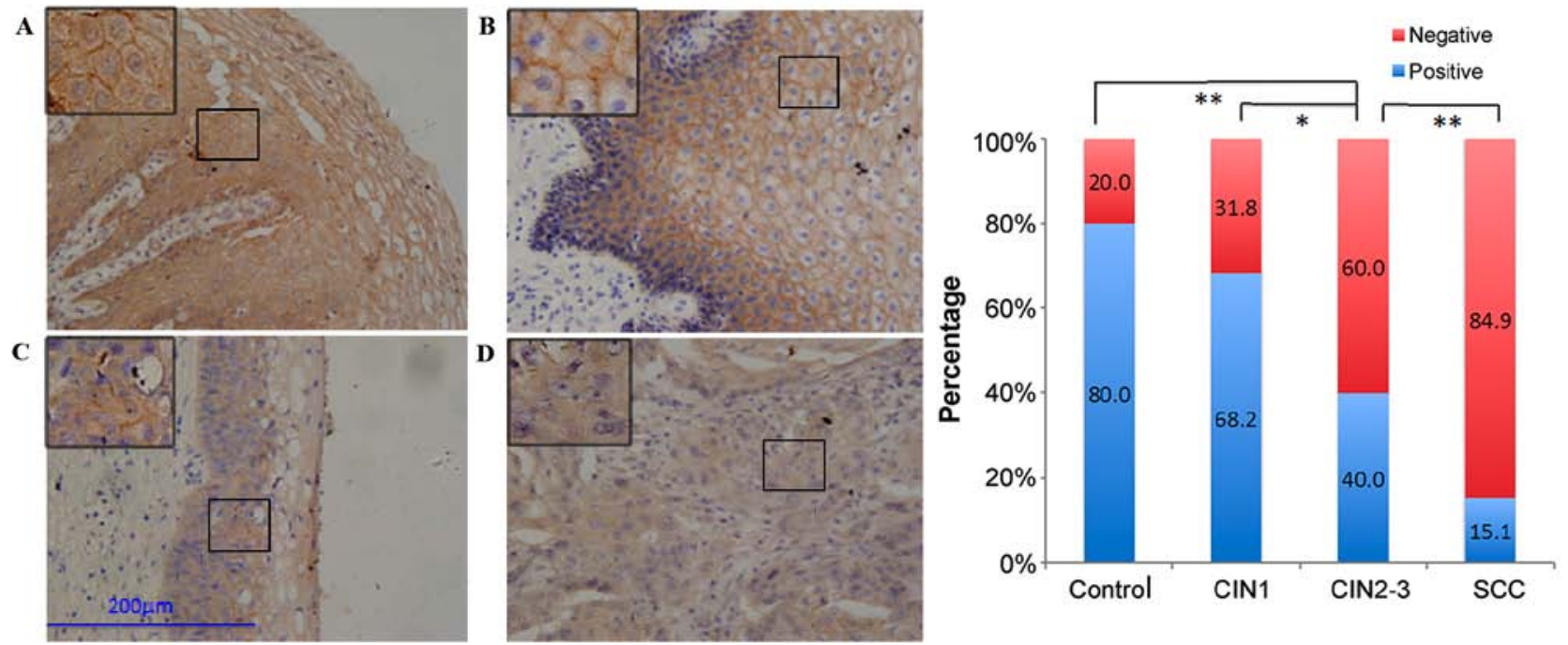

Figure 2. Immunohistochemical staining of $\beta$-catenin in cervical sections. Immunohistochemical staining of $\beta$-catenin in (A) normal human cervical tissue and (B) CIN1 (strong membranous staining), (C) CIN2-3 (decreased membranous staining and increased cytoplasmic staining), and (D) SCC (weak cytoplasmic staining). The scale bar is given at the left bottom. Insets are magnified images from selected areas (small squares). ${ }^{*} \mathrm{P}<0.05,{ }^{* *} \mathrm{P}<0.01$. CIN $1 / 2-3$, cervical intraepithelial neoplasia grade $1 / 2-3$; SCC, squamous cervical carcinoma.

positive relationships with HPV16 infection $\left(r_{s}=0.404,0.417\right.$, and 0.355 , respectively). Among early stage SCC cases, $81 / 86$ (94.2\%) had a HPV16 infection, but no significant relationship was indicated between HPV16 infection and the five EMT proteins $(\mathrm{P}>0.05)$.

\section{Discussion}

The precise involvement and mechanisms of HPV16 in EMT of CIN and SCC remain unknown. Therefore, the present study aimed to examine the expression of EMT indicators and their relationship with HPV16 in CIN and early stage SCC, and their prognostic value in early stage SCC. The results indicated that EMT occurs during the progression of CINs to early stage SCC, and is correlated to HPV16 infection in CINs. Lymph node metastasis and parametrial invasion are poor prognostic factors for early stage SCC, while positive E-cadherin expression may serve as a protective prognostic factor for early stage SCC.

E-cadherin is a calcium-dependent cell adhesion molecule serving a critical role among epithelial cells as its loss has been reported to contribute to cancer metastasis (33). During EMT, the downregulation of E-cadherin is a main indicator for intercellular junctions loss (20). $\beta$-catenin is a member of the catenin family and serves a vital role in cadherin adhesion functions (34). In the present study, it was indicated that the expression level of E-cadherin and $\beta$-catenin were reduced with disease progression, in a gradual manner. In addition, 

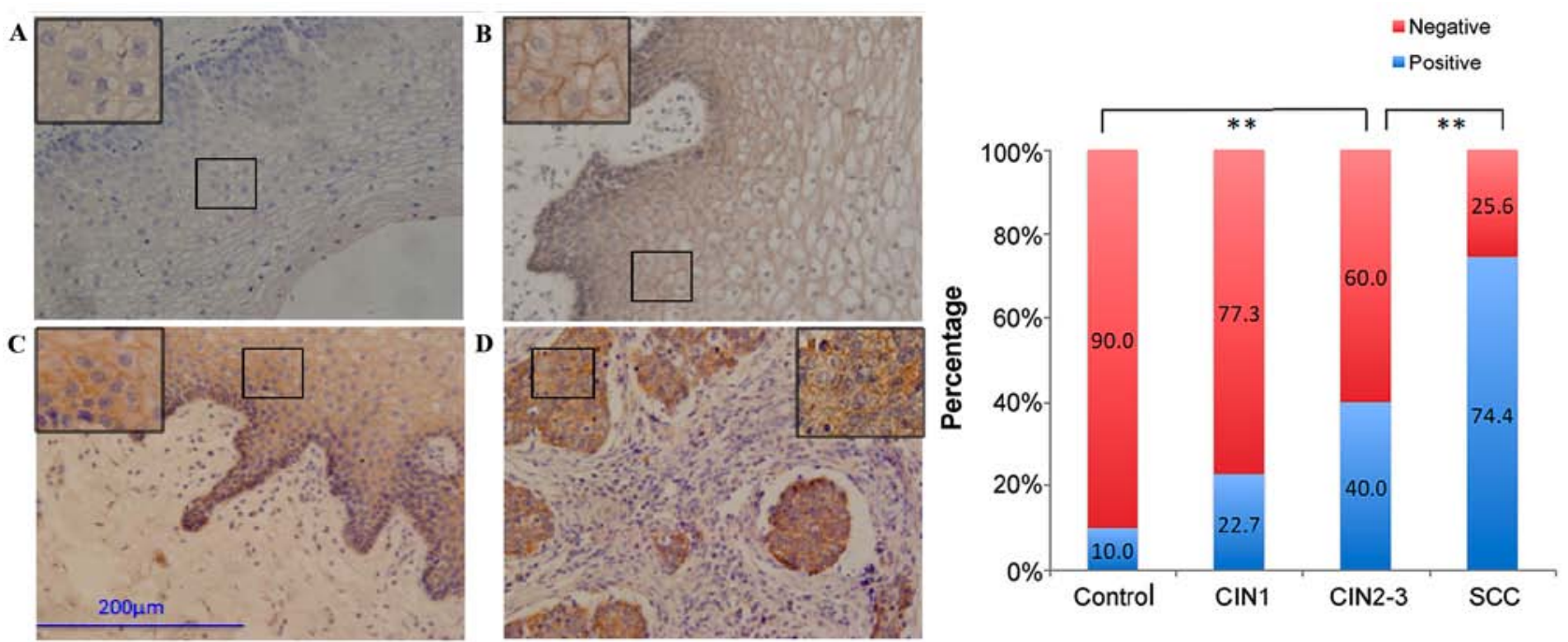

Figure 3. Immunohistochemical staining of $\mathrm{N}$-cadherin in cervical sections. Immunohistochemical staining of N-cadherin in (A) normal human cervical tissue (weak membranous staining), (B) CIN1 and (C) CIN2-3 (gradually increased membranous staining), and (D) SCC (strong membranous and cytoplasmic staining). The scale bar is given at the left bottom. Insets are magnified images from selected areas (small squares). ${ }^{* *} \mathrm{P}<0.01$. CIN $1 / 2-3$, cervical intraepithelial neoplasia grade 1/2-3; SCC, squamous cervical carcinoma.
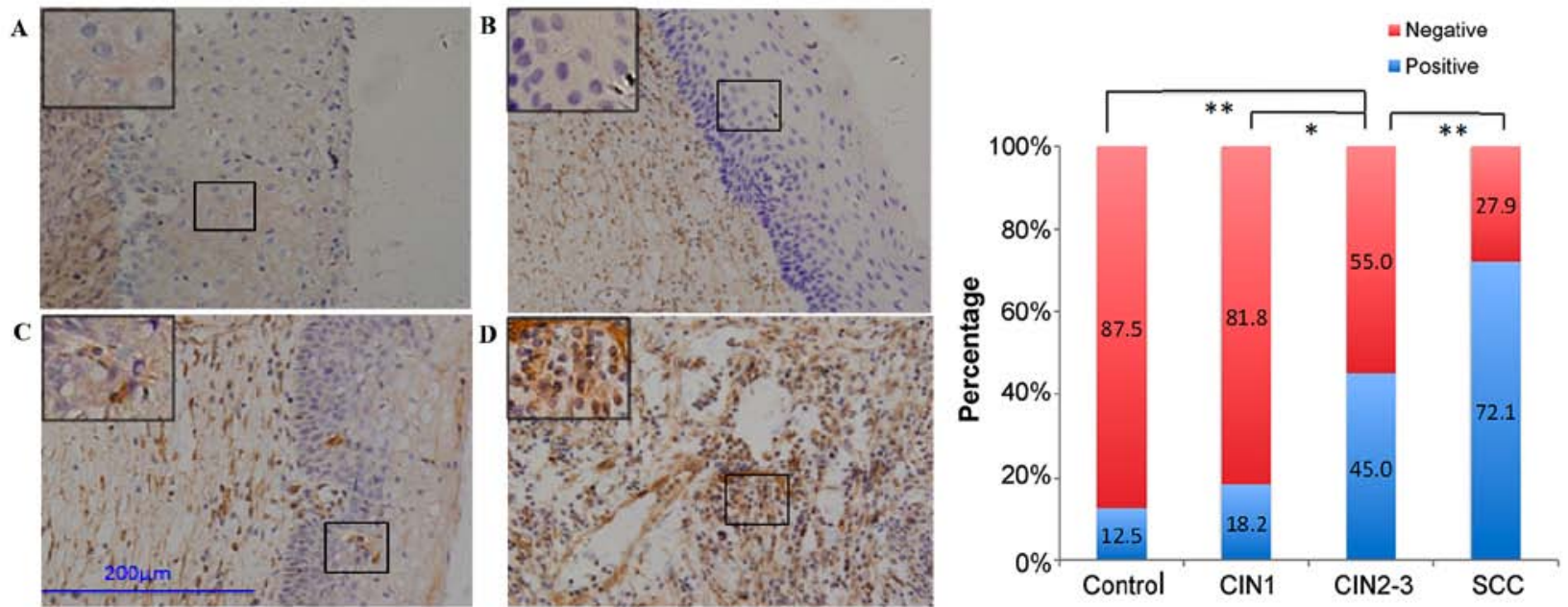

Figure 4. Immunohistochemical staining of vimentin in cervical sections. Immunohistochemical staining of vimentin in (A) normal human cervical tissue and (B) CIN1 (weak cytoplasmic staining), (C) CIN2-3 (moderate cytoplasmic staining), and (D) SCC (strong cytoplasmic and nuclear staining). The scale bar is given at the left bottom. Insets are magnified images from selected areas (small squares). ${ }^{*} \mathrm{P}<0.05,{ }^{* *} \mathrm{P}<0.01$. CIN1/2-3, cervical intraepithelial neoplasia grade $1 / 2-3$; SCC, squamous cervical carcinoma.

the expression pattern of $\beta$-catenin differed on a molecular level, as the expression level in the cell membrane declined, while its expression level in the cytoplasm was upregulated with the progression of cervical lesions. These results are in accordance to a previous study (35), in addition to the previous findings reporting that Wnt signaling inhibits the degradation process of $\beta$-catenin by phosphorylating and inhibiting GSK $3 \beta$ in tumors, thereby allowing $\beta$-catenin to accumulate in the cytosol and enter the nucleus, binding to the T-cell factor and driving transcription (36).

Accordingly, in the present study, the expression levels of E-cadherin and $\beta$-catenin in patients with early stage SCC with intravascular tumor thrombus and lymph node metastasis were significantly decreased compared with the expression levels in patients with SCC, but without tumor thrombus or lymph node metastasis. Therefore, from the aforementioned findings, it is suggested that downregulation of E-cadherin and $\beta$-catenin serves a role in the occurrence and development of SCC. Based on previous studies and the present results, reduced expression levels of E-cadherin and $\beta$-catenin are suggested to weaken intercellular adhesion, cause intercellular junction loss, morphological changes, and increase mobility of abnormal cells, leading to cancer metastasis $(6,8,11)$. In the present study, the expression of E-cadherin was a protective prognostic factor in patients with early stage SCC, supporting the concept that low EMT leads to a limited number of cells participating in the metastatic spread.

$\mathrm{N}$-cadherin and E-cadherin are members of the cadherin family, but they exhibit opposite effects, where E-cadherin mediates the adhesion between epithelial cells (33), as 

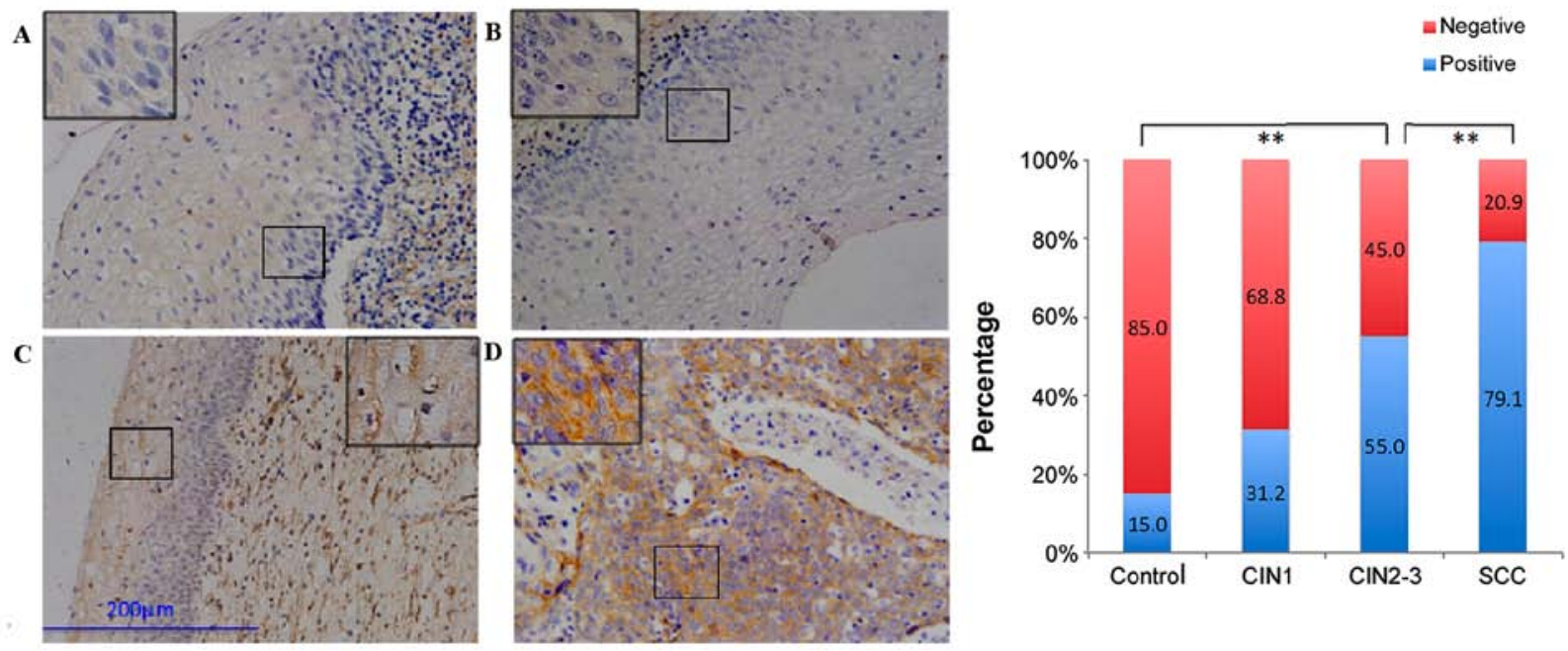

Figure 5. Immunohistochemical staining of fibronectin in cervical sections. Immunohistochemical staining of fibronectin in (A) normal human cervical tissue (weak cytoplasmic staining), (B) CIN1 and (C) CIN2-3 (gradually increased cytoplasmic staining), and (D) SCC (strong cytoplasmic staining). The scale bar is given at the left bottom. Insets are magnified images from selected areas (small squares). ${ }^{* *} \mathrm{P}<0.01$. CIN1/2-3, cervical intraepithelial neoplasia grade $1 / 2-3$; SCC, squamous cervical carcinoma.
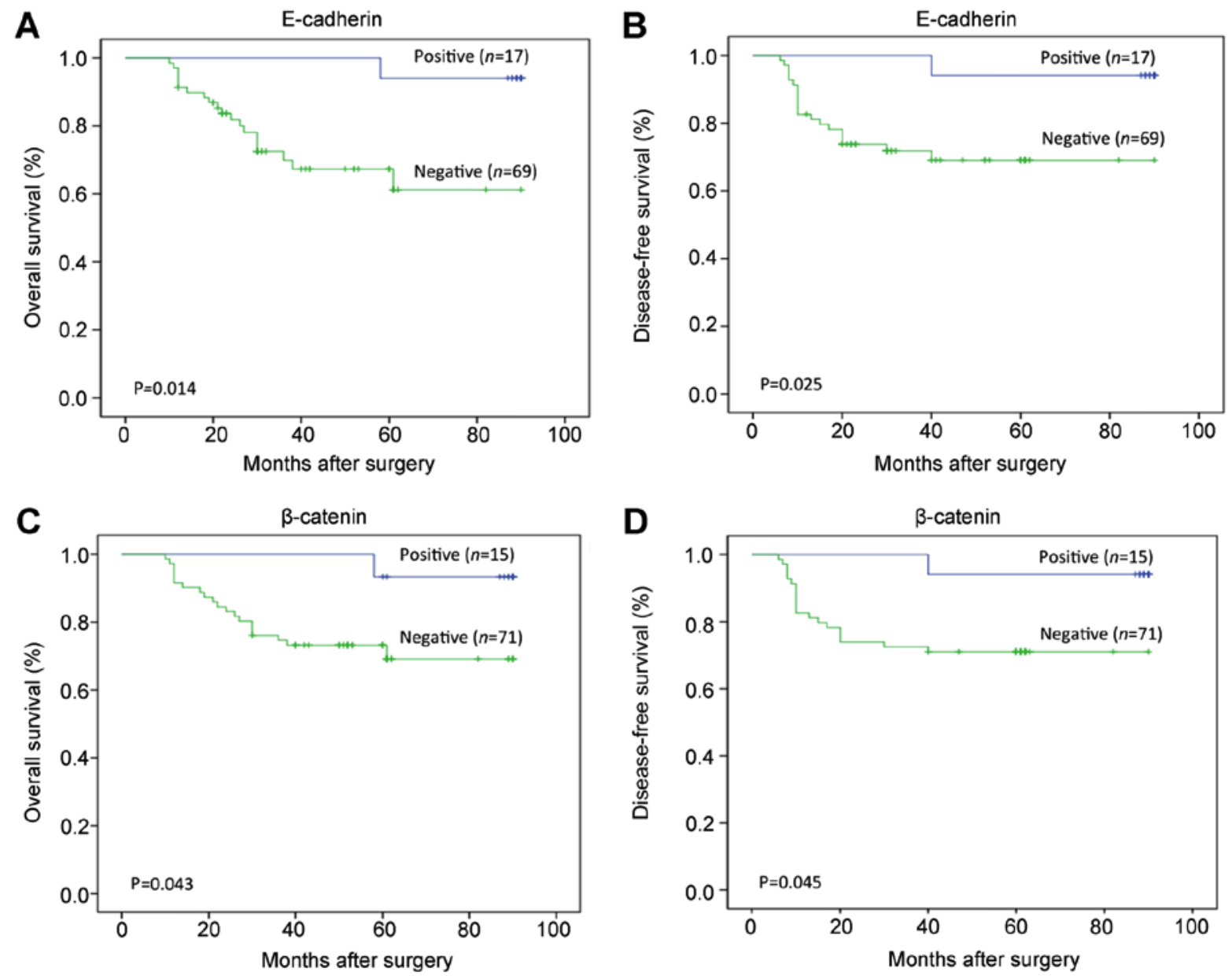

Figure 6. Overall survival and disease-free survival rates associated with E-cadherin and $\beta$-catenin expression. Kaplan-Meier analysis of (A) overall survival and (B) disease-free survival associated with the expression of E-cadherin. Kaplan-Meier analysis of (C) overall survival and (D) disease-free survival associated with the expression of $\beta$-catenin.

indicated above, and $\mathrm{N}$-cadherin promotes cell movement (37). The 'cadherin switch' is characterized by reduced expression of E-cadherin and increased expression of $\mathrm{N}$-cadherin and is believed to be one of the most important processes of EMT and its involvement in the metastatic spread of cancer cells (37). A number of studies have reported that abnormal 
Table II. Univariate survival analysis of factors associated with OS and DFS for patients with SCC.

\begin{tabular}{|c|c|c|c|c|c|c|}
\hline \multirow[b]{2}{*}{ Variables } & \multicolumn{2}{|c|}{$\mathrm{OS}$} & \multirow[b]{2}{*}{ P-value } & \multicolumn{2}{|c|}{ DFS } & \multirow[b]{2}{*}{ P-value } \\
\hline & HR & $95 \% \mathrm{CI}$ & & HR & $95 \% \mathrm{CI}$ & \\
\hline Age & 1.026 & $0.557-1.892$ & 0.934 & 1.055 & $0.311-3.583$ & 0.931 \\
\hline FIGO stage & 0.840 & $0.540-1.307$ & 0.840 & 0.676 & $0.280-1.633$ & 0.385 \\
\hline Histological grade & 1.385 & $0.891-2.154$ & 0.147 & 1.850 & $0.767-4.464$ & 0.171 \\
\hline Lymph node metastasis & 4.151 & $1.606-10.731$ & $0.003^{\mathrm{b}}$ & 4.043 & $1.567-10.432$ & $0.004^{\mathrm{b}}$ \\
\hline Parametrial invasion & 1.804 & $1.090-2.986$ & $0.022^{\mathrm{a}}$ & 1.744 & $1.055-2.883$ & $0.030^{\mathrm{a}}$ \\
\hline E-cadherin & 0.159 & $0.081-0.312$ & $<0.001^{\mathrm{b}}$ & 0.156 & $0.080-0.307$ & $<0.001^{\mathrm{b}}$ \\
\hline$\beta$-catenin & 0.575 & $0.422-0.784$ & $<0.001^{\mathrm{b}}$ & 0.332 & $0.179-0.616$ & $<0.001^{\mathrm{b}}$ \\
\hline $\mathrm{N}$-cadherin & 1.216 & $0.705-2.097$ & 0.481 & 1.491 & $0.502-4.433$ & 0.472 \\
\hline Vimentin & 1.689 & $0.915-3.116$ & 0.094 & 2.655 & $0.782-9.108$ & 0.118 \\
\hline Fibronectin & 1.662 & $0.802-3.445$ & 0.172 & 2.733 & $0.636-11.733$ & 0.176 \\
\hline
\end{tabular}

${ }^{\mathrm{a}} \mathrm{P}<0.05,{ }^{\mathrm{b}} \mathrm{P}<0.01$. OS, overall survival; DFS, disease-free survival; HR, hazard ratio; CI, confidence interval; SCC, squamous cervical carcinoma. HR $>1$ indicates that the risk for recurrence/mortality increased. HR $<1$ indicates that risk for recurrence/mortality decreased.
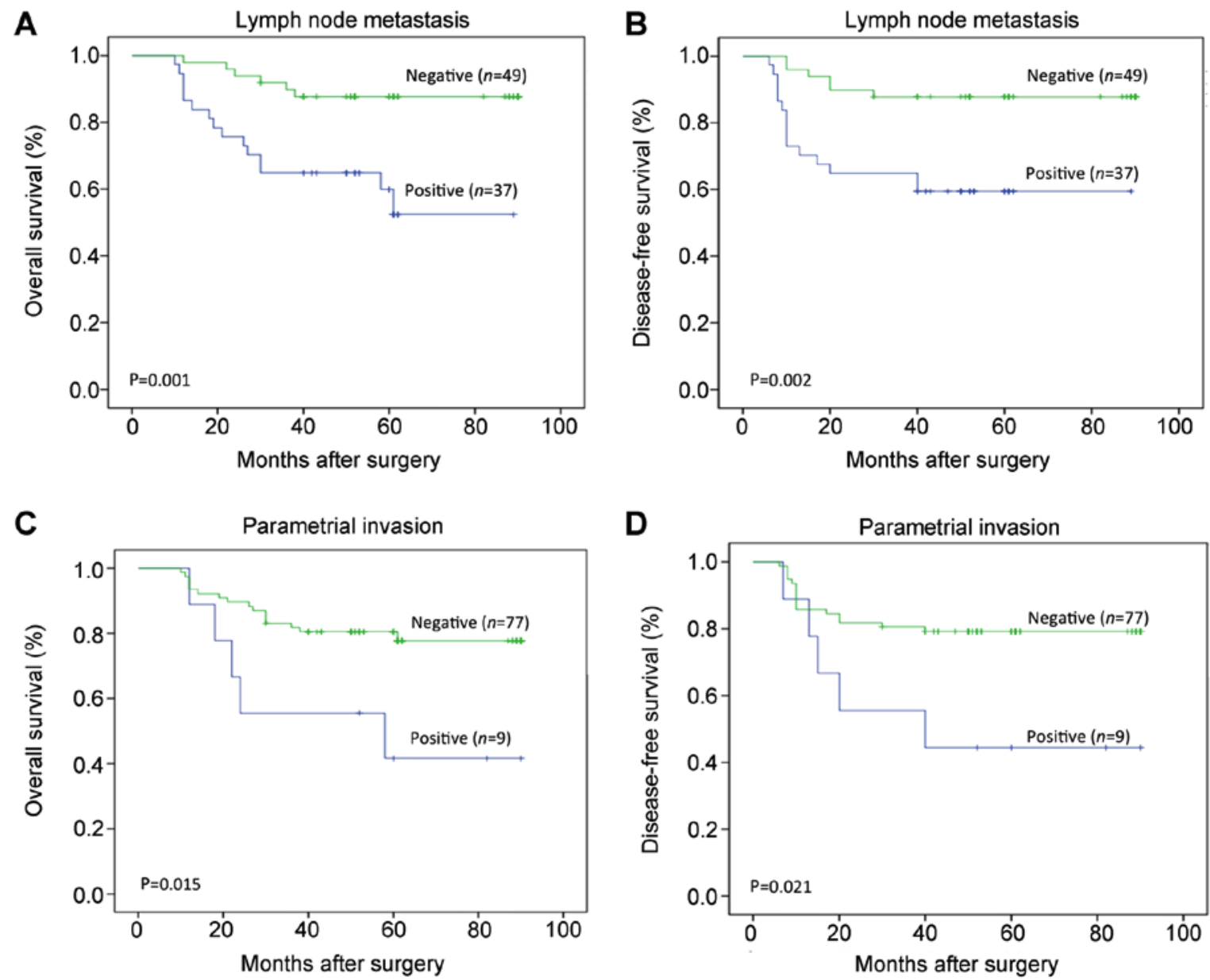

Figure 7. Overall survival and disease-free survival rates associated with lymph node metastasis and parametrial invasion. Kaplan-Meier analysis of (A) overall survival and (B) disease-free survival associated with lymph node metastasis. Kaplan-Meier analysis of (C) overall survival and (D) disease-free survival associated with parametrial invasion.

expression of $\mathrm{N}$-cadherin in epithelial tissues could promote morphological changes and EMT of cancer cells (38-41). In the present study, the expression level of $\mathrm{N}$-cadherin was very low in normal cervical tissues, in comparison to its expression level in the CIN tissues, while N-cadherin exhibited an even higher expression in SCC tissues. Its positive expression rate 
Table III. Multivariate survival analysis of OS and DFS in SCC patients.

\begin{tabular}{|c|c|c|c|c|c|c|}
\hline \multirow[b]{2}{*}{ Variables } & \multicolumn{2}{|c|}{ OS } & \multirow[b]{2}{*}{ P-value } & \multicolumn{2}{|c|}{ DFS } & \multirow[b]{2}{*}{ P-value } \\
\hline & HR & $95 \% \mathrm{CI}$ & & HR & $95 \% \mathrm{CI}$ & \\
\hline Lymph node metastasis & 3.544 & $1.361-9.280$ & $0.010^{\mathrm{a}}$ & 3.612 & $1.385-9.415$ & $0.009^{b}$ \\
\hline Parametrial invasion & 2.014 & $1.208-3.355$ & $0.007^{\mathrm{b}}$ & 1.935 & $1.165-3.214$ & $0.011^{\mathrm{a}}$ \\
\hline E-cadherin & 0.163 & $0.066-0.403$ & $<0.001^{\mathrm{b}}$ & 0.168 & $0.068-0.414$ & $<0.001^{\mathrm{b}}$ \\
\hline$\beta$-catenin & 0.611 & $0.176-2.120$ & 0.438 & 0.625 & $0.176-2.216$ & 0.466 \\
\hline
\end{tabular}

${ }^{\mathrm{a}} \mathrm{P}<0.05,{ }^{\mathrm{b}} \mathrm{P}<0.01$. OS, overall survival; DFS, disease-free survival; HR, hazard ratio; CI, confidence interval; SCC, squamous cervical carcinoma. HR $>1$ indicates that risk for recurrence/mortality increased; $H R<1$ indicates that risk for recurrence/mortality decreased.

Table IV. Correlations between HPV16 infection and expression of E-cadherin, $\beta$-catenin, $\mathrm{N}$-cadherin, vimentin, and fibronectin in CIN tissues $(\mathrm{n}=82)$.

\begin{tabular}{|c|c|c|c|c|}
\hline \multirow[b]{2}{*}{ Marker } & \multicolumn{2}{|c|}{ HPV16 } & \multirow[b]{2}{*}{$\mathrm{r}_{\mathrm{s}}$} & \multirow[b]{2}{*}{ P-value } \\
\hline & $\begin{array}{c}\text { Positive } \\
(n=27)\end{array}$ & $\begin{array}{c}\text { Negative } \\
(n=55)\end{array}$ & & \\
\hline E-cadherin & 6 & 37 & -0.424 & $<0.001^{\mathrm{a}}$ \\
\hline$\beta$-catenin & 13 & 26 & 0.008 & 0.941 \\
\hline $\mathrm{N}$-cadherin & 17 & 12 & 0.404 & $<0.001^{\mathrm{a}}$ \\
\hline Vimentin & 18 & 13 & 0.417 & $<0.001^{\mathrm{a}}$ \\
\hline Fibronectin & 20 & 20 & 0.355 & $0.001^{\mathrm{a}}$ \\
\hline
\end{tabular}

${ }^{\mathrm{a}} \mathrm{P}<0.01$. HPV16, human papilloma virus type 16 .

in the SCC group with parametrial invasion was significantly higher compared with the SCC group without parametrial invasion, and its positive expression rate in the group with lymph node metastasis was significantly higher compared with the group without metastasis. These results are supported by a previous study in nasopharyngeal carcinoma that indicated that the expression of $\mathrm{N}$-cadherin was correlated with lymph node metastasis (42). In conclusion, these findings suggest the important role of N-cadherin in SCC progression.

Vimentin (type III; $57 \mathrm{kD}$ ) is an intermediate filament that is found in the mesenchymal cells of various types of tissue during their developmental stages and maintains cell and tissue integrity (43). Increased abnormal expression of vimentin causes compositional changes in cytoskeletal proteins and transforms cuboidal epithelial cells into fusiform fibroblasts for easy migration $(44,45)$. It also leads to reoriented microtubule polarity and increased EMT phenotypes, due to increased $\beta 1$-integrin and the loss of junction protein E-cadherin (46). A number of studies have reported that vimentin was expressed abnormally in epithelial malignant tumors, including ovarian, breast, colon, esophageal, and prostate cancer (47-51).

Fibronectin is a non-collagen glycoprotein present in the extracellular matrix. Fibronectin regulates cell adhesion, proliferation, differentiation, and morphological maintenance of tissues under normal physiological conditions. It is associated with tumor invasion and metastasis in various pathological conditions $(52,53)$. In cervical lesions, fibronectin correlates positively with alpha $v$ beta 6 expression, an unfavorable prognostic factor of SCC (32). In the present study, vimentin and fibronectin exhibited a gradual increase in expression with the progression from CIN1 to CIN3, and expression significantly increased in SCC lesions. The expression levels of vimentin and fibronectin in the SCC group with tumor thrombi were significantly higher compared with the group without tumor thrombi. In addition, their expression levels in the group with lymph node metastasis were higher compared with the group without metastasis. These findings suggest that vimentin and fibronectin promote the progression of cervical lesions, and that their upregulated expression promotes the malignant-invasive behavior of SCC cells.

However, there was no indication that $\mathrm{N}$-cadherin, vimentin and fibronectin were independent prognostic factors of early stage SCC. This could be due to the fact that the effects of E-cadherin and $\beta$-catenin are stronger and may obscure the effects of N-cadherin, vimentin and fibronectin. Another possibility is that these factors are covariate. Additional studies are necessary to determine their exact prognostic significance in SCC. Nonetheless, there were no significant differences observed in the present study in the five EMT indicators among different SCC stages. This may be due to the relatively small sample size of the present study. In addition, EMT indicators may have a higher association with parametrial invasion and lymph node metastasis compared with FIGO stages. In the present study, lymph node metastasis and parametrial invasion were indicated to be poor prognostic factors for SCC. As these factors have already been identified in a number of studies (54-56), this suggests that the group of patients it the present study may be representative of the general population of patients with SCC.

The association between HPV16 infection and SCC occurrence has been extensively studied (22,57-59). HPV16, along with HPV18, was identified as an important factor to the development of cervical cancer in China in 2009 (60). A number of studies have confirmed that HPV16, similar to other tumor-causing viruses, including hepatitis B, Epstein-Barr virus and hepatitis $\mathrm{C}$, have the ability to downregulate E-cadherin and inhibit Langerhans cell adhesion and immune surveillance capability $(61,62)$, causing increased expression of $\mathrm{N}$-cadherin in epithelial cells (63), therefore, 
participating in EMT. The expression levels of E-cadherin in the cell membrane of HPV16-positive keratinocytes increased following the silencing of HPV16 E7 gene (64). This suggests that the HPV16 oncogene may lead to cervical lesions through cellular EMT. In the present study, 32.9\% (27/82) of the CIN cases had HPV16 infection, and HPV16 infection was negatively correlated with the expression level of E-cadherin and positively correlated with the expression level of $\mathrm{N}$-cadherin, vimentin, and fibronectin. The expression of E-cadherin gradually decreased, while the expression of $\mathrm{N}$-cadherin, vimentin, and fibronectin gradually increased with HPV16 infection in the progression of CIN lesions. Therefore, cervical epithelial cells may lose their morphology and characteristics during EMT and gain invasive properties, leading to the formation of progressive cervical lesions and, subsequently, SCC. There are a number of studies indicating the impact of the HPV E6 oncogene in the canonical Wnt/ $\beta$-catenin signaling pathway (65-67). E6 can augment the Wnt/ $\beta$-catenin/T cell factor (TCF) signaling response, however, it does not significantly alter $\beta$-catenin stability and expression (66). In addition, the ability of E6 to activate TCF response may be dependent, as well as independent, of $\beta$-catenin translocation (66). This may explain the lack of association of $\beta$-catenin and HPV16 in the present study.

However, in the present study no significant associations between HPV16 infection and the five EMT indicators were observed in the 86 cases of SCC. There are a number of reasons why this may have occurred, including the relatively small sample size of the present study. It may also be due to the fact that once HPV16 prompts CIN progression through EMT, it will affect SCC cells through other pathways, rather than EMT. Another possibility is that other oncogenic effects of HPV16 are stronger compared with EMT process. For example, HPV E6 and E7 oncogenes have the ability to degrade pRb, inactivate P53, interact with PDZ-containing proteins, regulate multiple epigenetic factors, encode miRNAs, to allow viral persistence, evade host immune surveillance, and deregulate cell cycle and apoptosis control $(68,69)$.

This was a single-center study and the number of patients was limited. In addition, the panel of proteins was limited. Further studies on a more comprehensive panel of factors potentially involved in EMT, invasion, and prognosis are required. In addition, in the present study only the protein levels were examined, therefore mRNA levels should also be examined in the future.

This is the first study, to the best of our knowledge, to systematically examine the expression of the five representative EMT markers in normal cervical tissue, CIN1, CIN2-3 and SCC. The expression of the epithelial markers E-cadherin and $\beta$-catenin reduced gradually, while the expression of the mesenchymal markers N-cadherin, vimentin, and fibronectin increased gradually during the progression of cervical squamous cell lesions. Patients with SCC with lymph node metastasis, parametrial invasion, negative E-cadherin, and negative $\beta$-catenin expression had shorter OS and DFS. Lymph node metastasis and parametrial invasion are independent poor prognostic factors, while positive E-cadherin expression may serve as an independent protective prognostic factor for early stage SCC. In addition, during the process of CIN, the rate of HPV16 infection was negatively correlated with the expression of E-cadherin and positively correlated with $\mathrm{N}$-cadherin, vimentin, and fibronectin. Therefore, HPV16 may cause CIN progression via an EMT-based pathway in cervical squamous epithelial cells. Nevertheless, the association between EMT and high-risk HPV require further investigation with a larger sample size of patients, in addition to the study of the underlying molecular mechanisms involved.

\section{Acknowledgements}

Not applicable.

\section{Funding}

The present study was supported by the National Natural Science Foundation of China (grant, no. 81101974).

\section{Availability of data and materials}

The datasets used and analyzed during the present study are available from the corresponding author on reasonable request.

\section{Authors' contributions}

JJ conceived the study, participated in study design and coordination, and assisted with drafting of the manuscript. XL performed the pathological study and drafted the manuscript. XY performed the clinical follow-up and the statistical analysis. JZ performed the histological examination and interpreted the data. BS contributed towards data analysis and the revision of the manuscript. All authors read and approved the final manuscript.

\section{Ethics approval and consent to participate}

The Institutional Review Board of The Second Hospital of Hebei Medical University (Hebei, China) approved the project. All methods were performed in accordance with the relevant guidelines and regulations. Written informed consent was received from all participants.

\section{Patient consent for publication}

Not applicable.

\section{Competing interests}

The authors declare that they have no competing interests.

\section{References}

1. Lertkhachonsuk AA, Yip CH, Khuhaprema T, Chen DS, Plummer M, Jee SH, Toi M and Wilailak S; Asian Oncology Summit 2013: Cancer prevention in Asia: Resource-stratified guidelines from the Asian Oncology Summit, 2013. Lancet Oncol 14: e497-e507, 2013.

2. Stanley M: Pathology and epidemiology of HPV infection in females. Gynecol Oncol 117 (2 Suppl): S5-S10, 2010.

3. Clifford GM, Smith JS, Plummer M, Muñoz N and Franceschi S: Human papillomavirus types in invasive cervical cancer worldwide: A meta-analysis. Br J Cancer 88: 63-73, 2003.

4. Torre LA, Bray F, Siegel RL, Ferlay J, Lortet-Tieulent J and Jemal A Global cancer statistics, 2012. CA Cancer J Clin 65: 87-108, 2015. 
5. Jiang J, Wei LH, Li YL, Wu RF, Xie X, Feng YJ, Zhang G, Zhao C, Zhao Y and Chen Z: Detection of TERC amplification in cervical epithelial cells for the diagnosis of high-grade cervical lesions and invasive cancer: A multicenter study in China. J Mol Diagn 12: 808-817, 2010.

6. Geiger TR and Peeper DS: Metastasis mechanisms. Biochim Biophys Acta 1796: 293-308, 2009.

7. Thiery JP, Acloque H, Huang RY and Nieto MA: Epithelial-mesenchymal transitions in development and disease. Cell 139: 871-890, 2009.

8. Guarino M, Rubino B and Ballabio G: The role of epithelial-mesenchymal transition in cancer pathology. Pathology 39: 305-318, 2007.

9. Zhau HE, Odero-Marah V, Lue HW, Nomura T, Wang R, Chu G, Liu ZR, Zhou BP, Huang WC and Chung LW: Epithelia to mesenchymal transition (EMT) in human prostate cancer: Lessons learned from ARCaP model. Clin Exp Metastasis 25: 601-610, 2008.

10. Yang MH, Chen CL, Chau GY, Chiou SH, Su CW, Chou TY, Peng WL and Wu JC: Comprehensive analysis of the independent effect of twist and snail in promoting metastasis of hepatocellular carcinoma. Hepatology 50: 1464-1474, 2009.

11. Gjerdrum C, Tiron C, Høiby T, Stefansson I, Haugen H, Sandal T, Collett K, Li S, McCormack E, Gjertsen BT, et al: Axl is an essential epithelial-to-mesenchymal transition-induced regulator of breast cancer metastasis and patient survival. Proc Natl Acad Sci USA 107: 1124-1129, 2010

12. Chambers AF, Groom AC and MacDonald IC: Dissemination and growth of cancer cells in metastatic sites. Nat Rev Cancer 2: 563-572, 2002

13. Guarino M: Epithelial-mesenchymal transition and tumour invasion. Int J Biochem Cell Biol 39: 2153-2160, 2007.

14. Greenburg G and Hay ED: Epithelia suspended in collagen gels can lose polarity and express characteristics of migrating mesenchymal cells. J Cell Biol 95: 333-339, 1982.

15. Thiery JP: Epithelial-mesenchymal transitions in development and pathologies. Curr Opin Cell Biol 15: 740-746, 2003.

16. Kowalski PJ, Rubin MA and Kleer CG: E-cadherin expression in primary carcinomas of the breast and its distant metastases. Breast Cancer Res 5: R217-R222, 2003.

17. Ngan CY, Yamamoto H, Seshimo I, Tsujino T, Man-i M, Ikeda JI, Konishi K, Takemasa I, Ikeda M, Sekimoto M, et al: Quantitative evaluation of vimentin expression in tumour stroma of colorectal cancer. Br J Cancer 96: 986-992, 2007.

18. Liang J, Zhou H, Peng Y, Xie X, Li R, Liu Y, Xie Q and Lin Z: $\beta$-catenin expression negatively correlates with WIF1 and predicts poor clinical outcomes in patients with cervical cancer. Biomed Res Int 2016: 4923903, 2016.

19. Lee MY, Chou CY, Tang MJ and Shen MR: Epithelial-mesenchymal transition in cervical cancer: Correlation with tumor progression, epidermal growth factor receptor overexpression, and snail up-regulation. Clin Cancer Res 14: 4743-4750, 2008.

20. Cheng Y, Zhou Y, Jiang W, Yang X, Zhu J, Feng D, Wei Y, Li M, Yao F, Hu W, et al: Significance of E-cadherin, $\beta$-catenin, and vimentin expression as postoperative prognosis indicators in cervical squamous cell carcinoma. Hum Pathol 43: 1213-1220, 2012

21. Li XL, Jiang J and Lu SY: Epithelial-mesenchymal transition and gynecologic oncology. Chin J Obstet Gynecol 47: 549-551, 2012 (In Chinese)

22. ChenX,Bode AM,DongZandCao Y: Theepithelial-mesenchymal transition (EMT) is regulated by oncoviruses in cancer. FASEB J 30: 3001-3010, 2016

23. Cyprian FS, Al-Farsi HF, Vranic S, Akhtar S and Al Moustafa AE: Epstein-barr virus and human papillomaviruses interactions and their roles in the initiation of epithelial-mesenchymal transition and cancer progression. Fron Oncol 8: 111, 2018.

24. Lapierre SG, Sauthier P, Mayrand MH, Dufresne S, Petignat P, Provencher D, Drouin P, Gauthier P, Dupuis MJ, Michon B, et al: Human papillomavirus (HPV) DNA triage of women with atypical squamous cells of undetermined significance with cobas $4800 \mathrm{HPV}$ and Hybrid Capture 2 tests for detection of high-grade lesions of the uterine cervix. J Clin Microbiol 50: 1240-1244, 2012.

25. Benedet JL, Bender H, Jones H III, Ngan HY and Pecorelli S: FIGO staging classifications and clinical practice guidelines in the management of gynecologic cancers. FIGO committee on gynecologic oncology. Int J Gynecol Obstet 70: 209-262, 2000.

26. Lax SF, Horn LC and Löning T: Categorization of uterine cervix tumors: What's new in the 2014 WHO classification. Pathologe 37: 573-584, 2016 (In German).
27. Isidean SD, Coutlée F and Franco EL: cobas 4800 HPV Test, a real-time polymerase chain reaction assay for the detection of human papillomavirus in cervical specimens. Expert Rev Mol Diagn 14: 5-16, 2014.

28. Jang TJ, Jung KH and Choi EA: Id-1 gene downregulation by sulindac sulfide and its upregulation during tumor development in gastric cancer. Int J Cancer 118: 1356-1363, 2006.

29. Fedchenko N and Reifenrath J: Different approaches for interpretation and reporting of immunohistochemistry analysis results in the bone tissue-a review. Diagn Pathol 9: 221, 2014.

30. Shi D, Jiang K, Fu Y, Fang R, Liu XI and Chen J: Overexpression of SPARC correlates with poor prognosis in patients with cervical carcinoma and regulates cancer cell epithelial-mesenchymal transition. Oncol Lett 11: 3251-3258, 2016.

31. Li B, Shi H, Wang F, Hong D, Lv W, Xie X and Cheng X: Expression of E-, $\mathrm{P}$ - and $\mathrm{N}$-cadherin and its clinical significance in cervical squamous cell carcinoma and precancerous lesions. PLoS One 11: e0155910, 2016.

32. Hazelbag S, Kenter GG, Gorter A, Dreef EJ, Koopman LA, Violette SM, Weinreb PH and Fleuren GJ: Overexpression of the alpha $\mathrm{v}$ beta 6 integrin in cervical squamous cell carcinoma is a prognostic factor for decreased survival. J Pathol 212: 316-324, 2007.

33. van Roy F and Berx G: The cell-cell adhesion molecule E-cadherin. Cell Mol Life Sci 65: 3756-3788, 2008.

34. Clevers H: Wnt/beta-catenin signaling in development and disease. Cell 127: 469-480, 2006

35. Stewart CJ and McCluggage WG: Epithelial-mesenchymal transition in carcinomas of the female genital tract. Histopathology 62: $31-43,2013$

36. Jeanes A, Gottardi CJ and Yap AS: Cadherins and cancer: How does cadherin dysfunction promote tumor progression? Oncogene 27: 6920-6929, 2008.

37. Hazan RB, Qiao R, Keren R, Badano I and Suyama K: Cadherin switch in tumor progression. Ann N Y Acad Sci 1014: 155-163, 2004.

38. Hazan RB, Phillips GR, Qiao RF, Norton L and Aaronson SA: Exogenous expression of $\mathrm{N}$-cadherin in breast cancer cells induces cell migration, invasion, and metastasis. J Cell Biol 148: 779-790, 2000

39. Lamouille S, Xu J and Derynck R: Molecular mechanisms of epithelial-mesenchymal transition. Nat Rev Mol Cell Biol 15: 178-196, 2014.

40. Costa LC, Leite CF, Cardoso SV, Loyola AM, Faria PR, Souza PE and Horta MC: Expression of epithelial-mesenchymal transition markers at the invasive front of oral squamous cell carcinoma. J Appl Oral Sci 23: 169-178, 2015.

41. Nakajima S, Doi R, Toyoda E, Tsuji S, Wada M, Koizumi M, Tulachan SS, Ito D, Kami K, Mori T, et al: N-cadherin expression and epithelial-mesenchymal transition in pancreatic carcinoma. Clin Cancer Res 10: 4125-4133, 2004

42. Sun H, Liu M, Wu X, Yang C, Zhang Y, Xu Z, Gao K and Wang F: Overexpression of $\mathrm{N}$-cadherin and $\beta$-catenin correlates with poor prognosis in patients with nasopharyngeal carcinoma. Oncol Lett 13: 1725-1730, 2017.

43. Coulombe PA and Wong P: Cytoplasmic intermediate filaments revealed as dynamic and multipurpose scaffolds. Nat Cell Biol 6: 699-706, 2004

44. Lang SH, Hyde C, Reid IN, Hitchcock IS, Hart CA, Bryden AA, Villette JM, Stower MJ and Maitland NJ: Enhanced expression of vimentin in motile prostate cell lines and in poorly differentiated and metastatic prostate carcinoma. Prostate 52: 253-263, 2002.

45. Singh S, Sadacharan S, Su S, Belldegrun A, Persad S and Singh G: Overexpression of vimentin: Role in the invasive phenotype in an androgen-independent model of prostate cancer. Cancer Res 63: 2306-2311, 2003.

46. Liu CY, Lin HH, Tang MJ and Wang YK: Vimentin contributes to epithelial-mesenchymal transition cancer cell mechanics by mediating cytoskeletal organization and focal adhesion maturation. Oncotarget 6: 15966-15983, 2015.

47. Colomiere M, Findlay J, Ackland L and Ahmed N: Epidermal growth factor-induced ovarian carcinoma cell migration is associated with JAK2/STAT3 signals and changes in the abundance and localization of alpha6betal integrin. Int J Biochem Cell Biol 41: 1034-1045, 2009

48. Vora HH, Patel NA, Rajvik KN, Mehta SV, Brahmbhatt BV, Shah MJ, Shukla SN and Shah PM: Cytokeratin and vimentin expression in breast cancer. Int J Biol Markers 24: 38-46, 2009.

49. Shirahata A, Sakata M, Sakuraba K, Goto T, Mizukami H, Saito M, Ishibashi K, Kigawa G, Nemoto H, Sanada Y and Hibi K: Vimentin methylation as a marker for advanced colorectal carcinoma. Anticancer Res 29: 279-281, 2009. 
50. Usami Y, Satake S, Nakayama F, Matsumoto M, Ohnuma K, Komori T, Semba S, Ito A and Yokozaki H: Snail-associated epithelial-mesenchymal transition promotes oesophageal squamous cell carcinoma motility and progression. J Pathol 215: 330-339, 2008.

51. Wei J, Xu G, Wu M, Zhang Y, Li Q, Liu P, Zhu T, Song A, Zhao L, Han Z, et al: Overexpression of vimentin contributes to prostate cancer invasion and metastasis via src regulation. Anticancer Res 28: 327-334, 2008.

52. Pankov R and Yamada KM: Fibronectin at a glance. J Cell Sci 115: 3861-3863, 2002 .

53. Birchler MT, Milisavlijevic D, Pfaltz M, Neri D, Odermatt B, Schmid S and Stoeckli SJ: Expression of the extra domain B of fibronectin, a marker of angiogenesis, in head and neck tumors. Laryngoscope 113: 1231-1237, 2003.

54. Huang L, Zheng M, Liu JH, Xiong Y, Ding H, Tang L and Wang HY: Risk factors and prognosis of IB-IIB cervical carcinoma with common iliac lymph node metastasis. Chin J Cancer 29: 431-435, 2010.

55. Li C, Liu W and Cheng Y: Prognostic significance of metastatic lymph node ratio in squamous cell carcinoma of the cervix. Onco Targets Ther 9: 3791-3797, 2016.

56. Liu Y, Zhao LJ, Li MZ, Li MX, Wang JL and Wei LH: The number of positive pelvic lymph nodes and multiple groups of pelvic lymph node metastasis influence prognosis in stage IA-IIB cervical squamous cell carcinoma. Chin Med J (Engl) 128 : 2084-2089, 2015.

57. Ajila V, Shetty H, Babu S, Shetty V and Hegde S: Human papilloma virus associated squamous cell carcinoma of the head and neck. J Sex Transm Dis 2015: 791024, 2015.

58. Bulk S, Berkhof J, Bulkmans NW, Zielinski GD, Rozendaal L, van Kemenade FJ, Snijders PJ and Meijer CJ: Preferential risk of HPV16 for squamous cell carcinoma and of HPV18 for adenocarcinoma of the cervix compared to women with normal cytology in The Netherlands. Br J Cancer 94: 171-175, 2006.

59. Cerasuolo A, Annunziata C, Tortora M, Starita N, Stellato G Greggi S, Maglione MG, Ionna F, Losito S, Botti G, et al: Comparative analysis of HPV16 gene expression profiles in cervical and in oropharyngeal squamous cell carcinoma. Oncotarget 8: 34070-34081, 2017.
60. Chen W, Zhang X, Molijn A, Jenkins D, Shi JF, Quint W, Schmidt JE, Wang P, Liu YL, Li LK, et al: Human papillomavirus type-distribution in cervical cancer in China: The importance of HPV 16 and 18. Cancer Causes Control 20: 1705-1713, 2009.

61. Laurson J, Khan S, Chung R, Cross K and Raj K: Epigenetic repression of E-cadherin by human papillomavirus $16 \mathrm{E} 7$ protein. Carcinogenesis 31: 918-926, 2010.

62. Cheng YM, Chou CY, Hsu YC, Chen MJ and Wing LY: The role of human papillomavirus type $16 \mathrm{E} 6 / \mathrm{E} 7$ oncoproteins in cervical epithelial-mesenchymal transition and carcinogenesis. Oncol Lett 3: 667-671, 2012.

63. Hellner K, Mar J, Fang F, Quackenbush J and Munger K: HPV16 E7 oncogene expression in normal human epithelial cells causes molecular changes indicative of an epithelial to mesenchymal transition. Virology 391: 57-63, 2009.

64. Caberg JH, Hubert PM, Begon DY, Herfs MF, Roncarati PJ, Boniver JJ and Delvenne PO: Silencing of E7 oncogene restores functional E-cadherin expression in human papillomavirus 16-transformed keratinocytes. Carcinogenesis 29: 1441-1447, 2008.

65. Yang $M$, Wang M, Li X, Xie Y, Xia X, Tian J, Zhang K and Tang A: Wnt signaling in cervical cancer? J Cancer 9: 1277-1286, 2018.

66. Bello JO, Nieva LO, Paredes AC, Gonzalez AM, Zavaleta LR and Lizano M: Regulation of the Wnt/ $\beta$-catenin signaling pathway by human papillomavirus E6 and E7 oncoproteins. Viruses 7: 4734-4755, 2015

67. Sominsky S, Kuslansky Y, Shapiro B, Jackman A, Haupt Y, Rosin-Arbesfeld R and Sherman L: HPV16 E6 and E6AP differentially cooperate to stimulate or augment Wnt signaling. Virology 468-470: 510-523, 2014.

68. Ghittoni R, Accardi R, Chiocca S and Tommasino M: Role of human papillomaviruses in carcinogenesis. Ecancermedicalscience 9: $526,2015$.

69. Yeo-Teh NSL, Ito Y and Jha S: High-risk human papillomaviral oncogenes E6 and E7 target key cellular pathways to achieve oncogenesis. Int J Mol Sci 19: pii: E1706, 2018. 